\title{
Light-mediated activation reveals a key role for Rac in collective guidance of cell movement in vivo
}

\author{
Xiaobo Wang ${ }^{1,{ }^{*}}$, Li He ${ }^{1, *}$, Yi I. Wu ${ }^{2}$, Klaus M. Hahn ${ }^{2}$, and Denise J. Montell ${ }^{1,{ }^{*}}$ \\ ${ }^{1}$ Department of Biological Chemistry Center for Cell Dynamics Johns Hopkins School of Medicine \\ 855 North Wolfe Street Baltimore, MD 21205 USA \\ ${ }^{2}$ Department of Pharmacology Lineberger Comprehensive Cancer Center University of North \\ Carolina, Chapel Hill Chapel Hill, North Carolina 27599 USA
}

\begin{abstract}
The small GTPase Rac induces actin polymerization, membrane ruffling, and focal contact formation in cultured single cells1, but can either repress or stimulate motility in epithelial cells depending on the conidtions 2,3 . Therefore the role of Rac in collective epithelial cell movements in vivo, which are important for both morphogenesis and metastasis4-7, is difficult to predict. Recently photoactivatable analogs of Rac (PA-Rac) have been developed, allowing rapid and reversible activation or inactivation of Rac using light8. In cultured single cells, light-activated Rac leads to focal membrane ruffling, protrusion, and migration. Here we show that focal activation of Rac is also sufficient to polarize an entire group of cells in vivo, specifically the border cells of the Drosophila ovary. Moreover activation, or inactivation, of Rac in one cell of the cluster caused a dramatic response in the other cells, suggesting that the cells sense direction as a group based on relative levels of Rac activity. Communication between cells of the cluster required Jun N-terminal kinase (JNK) but not guidance receptor signaling. These studies further show that photoactivatable proteins are effective tools in vivo.

Border cells are a group of 6-8 cells that arise from the monolayer of $\sim 650$ epithelial follicle cells that surround 15 nurse cells and one oocyte in a structure called an egg chamber (Figure 1a-c). Border cells migrate $\sim 175 \mu \mathrm{m}$ in between the nurse cells, as an interconnected group of two distinct cell types: 4-8 migratory cells surround two central polar cells (Figure $1 \mathrm{~d}-\mathrm{i}, \mathrm{k})$. Polar cells cannot migrate but secrete a cytokine that activates the JAK/STAT pathway rendering the outer cells motile9. The outer cells carry the polar cells and lose the ability to move in the absence of continuous JAK/STAT activation10. Thus each cell type requires the other. Border cells also require steroid hormone, receptor tyrosine kinase, Notch, and other signaling cascades11-16. Thus border cells experience a rich and complex signaling environment, as do most cells in vivo.
\end{abstract}

\footnotetext{
Users may view, print, copy, download and text and data- mine the content in such documents, for the purposes of academic research, subject always to the full Conditions of use: http://www.nature.com/authors/editorial_policies/license.html\#terms

${ }^{3}$ To whom correspondence should be addressed dmontell@jhmi.edu.

These authors contributed equally

Author contributions X. W. carried out the experiments documented in Figures 1-3 and S1-9. Li He carried out the experiments shown in Figures 4-5 and S10-12. X.W. made all of the transgenic flies and helped Li to collect FRET data in Figure 5. Y.W. developed and provided the PA-Rac constructs and advised X. W. on their use. K.M.H. and D.J.M. coordinated the study. D.J.M. prepared the final version of the manuscript, based on contributions from all authors.
} 
The requirement for Rac in border cell migration was one of the earliest demonstrations of its role in cell motility in vivo17. Expression of either dominant-negative or constitutively active Rac impedes migration13,17, 18, suggesting that its activity must be spatially and/or temporally controlled. However the precise function of Rac remains unclear.

To evaluate the effect of locally activating Rac in border cells we generated transgenic flies expressing the photoactivatable form of Rac (PA-RacQ61L) tagged with mCherry, under control of the Gal4/UAS system. When expressed in border cells using slbo-Gal4, the protein was distributed throughout the cells, in the cytoplasm, nuclei, and at cell surfaces (Figure 1g-i). In the absence of laser illumination, border cell migration was normal (Figure S1; Movies S1 and S2).

Upon exposure to repeated pulses of laser light, border cell migration could be redirected (Figure 11-t; Movie S3). In this example, border cells were migrating along the path designated by the solid arrow and the leading cell extended a prominent forward-directed protrusion. The laser was applied to the cell next to the leading cell, which did not exhibit any detectable protrusion at the time. Following illumination the cluster retracted the original forward protrusion, changed direction and began moving to the side, a behavior never observed in wild-type19, 20. Light pulses were delivered once per minute due to the reversibility of PA-Rac8. The border cells reached the side of the egg chamber after $\sim 60$ minutes (Figure 1m,n and Movie S3). Although light pulses were continuously delivered, the cluster did not move further down the side of the egg chamber over the next 20 minutes (Figure 1o-q), suggesting there might be a barrier or repellent in this region. When we shifted the site of illumination toward the center of the egg chamber (Figure 1r), the cells responded by moving in that direction (Figure 1s,t; Movie S3). A single amino acid substitution in the LOV domain $(\mathrm{C} 450 \mathrm{M})$ renders the protein light-insensitive8 and this construct could not redirect border cell migration even in the presence of light (Figure 1u-w; Movie S4).

To determine if Rac activity was only required in the lead cell, we co-expressed dominantnegative Rac (RacT17N) together with PA-RacQ61L in all border cells and photoactivated Rac in one cell. RacT17N alone strongly inhibits border cell motility 18 and photoactivation of Rac in the front cell failed to promote forward movement of the cluster in this background (Figure 1g-i). However, activating Rac in approximately half of the cells in the cluster caused them to move forward, albeit very slowly (Figure $1 \mathrm{j}-\mathrm{m}$ ). These results suggest that each cell requires some Rac activity for motility, and each cell contributes to the migration speed of the cluster, but the highest level of Rac activation determines the direction of movement.

We then tested whether PA-RacQ61L was sufficient to cause border cells to move in a direction opposite to their normal movement (Figure 2). Border cells expressing PARacQ61L were first driven in the normal direction, and prominent lamellipodia-like protrusion was evident at the site of illumination (Movie S5). Then, we illuminated the rear. Front protrusion ceased rapidly (Movie S5) but rearward movement was initially very slow. After a variable delay, clusters moved backwards (Figure 2d-i), sometimes reconnecting with a follicle cell within the epithelium (Movie S5). In contrast, the light-insensitive control 
protein did not reverse the migration direction (Movie S6). On average, the PA-Rac-induced forward migration speed exceeded the reverse migration speed by 4.5 -fold (Figure 2p; PARac Front vs. PA-Rac Back), suggesting an influence of endogenous directional signaling on the behavior induced by PA-Rac.

To explore the interaction between endogenous signals and PA-Rac, we compared the responses of wild-type cells to those of cells with reduced guidance receptor activity. PVR and EGFR are receptor tyrosine kinases that function redundantly to guide migrating border cells13-15. Border cells expressing dominant-negative forms of both guidance receptors, $\mathrm{PVR}^{\mathrm{DN}}$ and $\mathrm{EGFR}^{\mathrm{DN}}$, extend protrusions in all directions and make little forward progress20. PA-Rac rescued both the morphological defect and directional movement in this genotype (Figure 2j-1), consistent with the idea that Rac normally functions downstream of the receptors to determine the direction of movement. When clusters were illuminated at the front, the cells moved forward (Figure 2j-1). When the same clusters were illuminated at the back, rearward movement (Figure $2 \mathrm{~m}$-o ) resulted. In contrast to the responses of wild-type clusters, average forward and reverse migration speeds were indistinguishable in border cells expressing $\mathrm{PVR}^{\mathrm{DN}}$ and $\mathrm{EGFR}^{\mathrm{DN}}$ (Figure 2p), supporting the idea of competition between endogenous guidance receptor signaling and PA-Rac induced directionality.

After stimulating rearward protrusion, we stopped illuminating and observed the recovery (Figure S2). Both wild-type and $\mathrm{PVR}^{\mathrm{DN}}$ - and EGFR ${ }^{\mathrm{DN}}$-expressing clusters rapidly protruded in response to rear illumination and retracted the rearward protrusion following cessation of the light. However, wild-type cells protruded less and retracted more (Figure S2q). Over longer time courses, wild-type cells typically stalled after cessation of rear illumination but eventually recovered movement in the normal forward direction (Figure S3a-m). In contrast, $\mathrm{PVR}^{\mathrm{DN}}$ and $\mathrm{EGFR}^{\mathrm{DN}}$-expressing clusters failed to recover forward movement (Figure S3m-y). These results also suggest that endogenous PVR and EGFR signals compete with PA-RacQ61L-induced polarization.

The inability of PA-RacQ61L to cause border cells to move down the side of the egg chamber led us to probe the microenvironment further. Within the anterior $\sim 1 / 3$ of their normal travel path, focal Rac activation could steer border cells from the center path all the way to the follicle cells, or along the perimeter of the egg chamber (Figure 3a-c and $\mathrm{m}$ ). However if we treated cells after they reached the center of the egg chamber, they could not be redirected to the follicle cell layer (Figure 3d-i and m), although PA-RacQ61L could still move them forwards or backwards. Within the posterior $1 / 3$ of their normal path, the cells could again be directed off their normal course, in between the nurse cells (Figure 3j-1 and $\mathrm{m})$. A summary of the responses to PA-RacQ61L is shown in Figure $3 \mathrm{~m}$. Thus there are regions in the egg chamber that actively repel the border cells or lack important structural or chemical substrates for migration, suggesting that there is additional guidance information besides the ligands for PVR and EGFR.

PA-RacQ61L was also insufficient to cause border cells to migrate earlier than normal, possibly because high levels of JAK/STAT signaling, which are required for the border cells to initiate movement, are not achieved at earlier time points21, 22. Consistent with this, PARacQ61L did not cause protrusion or migration in border cells expressing a dominant- 
negative form of the receptor Domeless, which is required for STAT activation (Figure S4af). A key downstream target of STAT is the transcription factor Slow Border Cells (SLBO)23, and slbo mutant border cells cannot extend protrusions. However PA-RacQ61L could not rescue protrusion or migration in slbo mutants (Figure S4g-1). PA-RacQ61L also failed to rescue guidance receptor deficiency after stage 10 (not shown). Together these findings demonstrate that PA-RacQ61L reveals temporal as well as spatial constraints on migrating cells.

To evaluate the effects of locally inhibiting Rac, we generated transgenic flies expressing PA dominant-negative Rac (UAS-PA-RacT17N). Illuminating the leading border cell arrested migration and, strikingly, led to protrusion at the cluster rear (Figure S5a-j; Movie S7). In contrast illumination of the rear of the cluster enhanced forward protrusion (Figure S5k-m) and migration (Figure 2p). The magnitude of the effect was smaller in PVR ${ }^{\mathrm{DN}}$ and EGFR $^{\text {DN }}$-expressing cells (Figure S5n-p; Figure 2p).

The non-autonomous effects of PA-RacQ61L and PA-RacT17N were striking so we examined the morphological consequences at higher magnification. Specifically, activation of Rac in one cell of either a wild-type cluster (Figure 4a-c) or a cluster expressing PVR ${ }^{\mathrm{DN}}$ and $\mathrm{EGFR}^{\mathrm{DN}}$ (Figure $4 \mathrm{~d}-\mathrm{f}$ ) resulted in retraction of protrusions by the other cells and movement of the cluster in the direction of the light. This was true whether the illumination was provided at the front of the cluster (not shown) or at the back. Strikingly PA-RacT17N had precisely the opposite effect in a polarized wild-type cluster (Figure $4 \mathrm{j}-1)$. Focal inhibition of Rac in the protruding lead cell caused a loss of polarization and random protrusion of all the cells in the cluster (Figure 41).

To quantify these results, we developed an automated method to count the number of protruding cells (Figure S6) and calculated the directionality index, which measures the degree of polarization of the cell cluster20. PA-RacQ61L treatment rescued the $\mathrm{PVR}^{\mathrm{DN}}, \mathrm{EGFR}^{\mathrm{DN}}$ polarization and the number of protruding cells nearly to wild-type (Figure $4 m, n$ ).

Inhibition of the JNK pathway also reduces the directionality index 24 (Figure 4n). The JNK pathway helps to coordinate border cell movement by promoting cohesion between border cells. To test the hypothesis that cluster cohesion is important for the non-autonomous effects of Rac, we monitored the effect of PA-RacQ61L in cells with reduced JNK signaling. Photoactivation of Rac at the back of clusters with impaired JNK signaling did not cause retraction of protrusions that were extended in other directions and resulted in little net movement of the cluster (Figure 4g-i). The same effect was observed whether JNK signaling was reduced by expression of the JNK phosphatase Puckered (UAS-Puc2A) or by expressing a dominant-negative form of the kinase (not shown).

The inability of PA-RacQ61L to rescue the JNK knockdown phenotype could have been because JNK signaling is required autonomously downstream of Rac to generate lamellipodial protrusion. However PA-RacQ61L induced autonomous cell protrusion in the direction of illumination, even in cells over-expressing puc2A (Figure 4g-i). Therefore JNK signaling is not required downstream of Rac to promote protrusion, consistent with the 
published observation that reduction of JNK signaling does not lead to reduced protrusion24. Together these results suggest that JNK signaling is required for the nonautonomous propagation of directional information from the cell with highest Rac activity to the other cells of the cluster. This could be due to direct mechanical coupling of the cells or via signaling pathways downstream of adhesion receptors or both.

Our results suggested that Rac is normally active in all the cells of the cluster, that the leading cell has a higher level of Rac activity, and this asymmetry is lost in $\mathrm{PVR}^{\mathrm{DN}}$ - and EGFR $^{\mathrm{DN}}$-expressing cells. To test this we took advantage of a Rac fluorescence resonance energy transfer (FRET) biosensor25. When expressed in Drosophila S2 cells, biosensor activity increased in response to EGF stimulation, and the increase was blocked by coexpression of dominant-negative Rac (Figure S7a-k). We generated transgenic flies expressing the biosensor under the control of Gal4/UAS. When expressed with slbo-Gal4, we consistently observed a FRET signal in border cells (Figure S71,m), and this was dramatically reduced upon co-expression of dominant-negative Rac (Figure S7n,o). Moreover the signal within the border cell cluster was asymmetric and appeared highest in elongating protrusions, which were most prominent in the leading cell (Figure 5a-e). This FRET signal was inhibited by co-expression of RacT17N (Figure 5l). To quantify the asymmetry we divided the border cell cluster into 30 sectors (where sector 0 represents the front of the cluster and -15 and +15 represent the rearmost sector), and measured the FRET efficiency in each sector for more than 30 clusters (Figure $5 \mathrm{f}-\mathrm{g}$ ). As predicted, the Rac activity was highest at the front (between sectors -5 and +5 ) and lowest at the back (Figure $5 \mathrm{~h}, \mathrm{i}, \mathrm{m})$. We then measured the Rac activity in more than 30 border cell clusters expressing $\mathrm{PVR}^{\mathrm{DN}}$ and EGFR ${ }^{\mathrm{DN}}$ and found no difference between front and back (Figure j,k,m), consistent with the proposal that asymmetric Rac activation requires guidance receptor input. In the absence of such asymmetry, non-directional signals activate Rac uniformly, stimulating random protrusion.

During normal morphogenesis and in tumor metastasis, many cells move in interconnected groups in a process termed collective cell migration4-7. Border cells represent one model for the study of such movements. We previously found that guidance receptor signaling not only promotes border cell protrusion at the front of the cluster but also polarizes the group so as to inhibit protrusion at the rear20. However it was unclear to what extent each cell sensed direction independently or whether they did so collectively and what intracellular signal(s) downstream of the receptors would be sufficient to polarize the group26. The results presented here demonstrate that a local increase in Rac activity is sufficient not only to stimulate protrusion autonomously in the treated cell but also to cause retraction of side and back cells, resulting in net cluster polarization and movement in the direction of highest Rac activity. Conversely inhibition of Rac in the lead cell caused the other cells to protrude in all directions as if guidance receptor activity were lost. These results suggest that elevated guidance activity at the front of the cluster activates Rac to a higher level in the front cell and this is sufficient to set the direction of migration for the whole group. Despite the fact that receptor tyrosine kinases activate myriad downstream signaling pathways, other pathways do not appear to be necessary, though they may play redundant or overlapping roles. Thus, asymmetric Rac activity is key for direction-sensing in vivo. We also show that 
JNK signaling is required to transmit the guidance signal between cells of the cluster. This work further suggests that photoactivatable proteins are likely to be a powerful new class of tools for the manipulation of protein activities with fine spatial and temporal control to address a variety of biological questions in animals.

\section{Methods Summary \\ Drosophila strains}

New transgenic fly lines were generated by Bestgene Inc. N-terminal-cherry tagged PARacQ61L, PA-RacT17N, the light insensitive control C450M-PA-RacQ61L8 and the Rac FRET probe were inserted into pUASt Drosophila expression vector using the Gateway recombination system (Invitrogen). P[slbo-GALA]27 drives UAS transgene expression in outer, migratory border cells but not polar cells even though the endogenous slbo gene and protein product are expressed in both cell types23. P[UAS-MCD8-GFP]28, P[UAS-moesinGFP $] 29, P[U A S-D R a c T 17 N]$ and P[UAS-DRacV12]30 have been described previously. $P\left[U A S-P V R^{D N}\right]$ and $P\left[U A S-E G F R^{D N}\right]$ were obtained from P. Rørth13. P[UAS-Puc2A] and $P[U A S-D n B s k]$ were obtained from E. Martin-Blanco24. All stocks were maintained at room temperature. Before dissection, flies were maintained at $29^{\circ} \mathrm{C}$ overnight to increase trangene expression levels. This incubation had no negative effect on border cell migration.

\section{Imaging and photomanipulation}

Drosophila egg chambers were dissected and mounted in Schneider's insect medium supplemented with $20 \% \mathrm{FBS}$ and $0.10 \mathrm{mg} / \mathrm{ml}$ insulin as described19, 20. Photoactivation, time-lapse-imaging, and 3D morphological reconstruction were carried out using a Zeiss 510-Meta confocal microscope using a 63X, 1.4 numerical aperture lens with $2 \mathrm{X}$ zoom. To photoactivate, the $458 \mathrm{~nm}$ laser was set at $10 \%$ power for $0.1 \mathrm{~ms}$ per pixel in a $7 \mu \mathrm{m}$ spot and the photoactivation scan took approximately 25 seconds. After 30 seconds, border cells were imaged using $568 \mathrm{~nm}$. This series of steps was repeated for the duration of the timelapse experiment. Where indicated, $15-20 \mathrm{Z}$ planes separated by $1.5 \mu \mathrm{m}$ were obtained before and after photoactivation (samples were illuminated every 80 seconds for one hour). 3D reconstructions were rendered using Imaris software.

S2 cells were transfected with the Rac FRET vector with or without the Rac ${ }^{\mathrm{DN}}$ vector using the QIAGEN Effectine Kit. Cells were transferred to serum-free medium $48 \mathrm{hrs}$ after transfection and cultured for another $6 \mathrm{hrs}$. Then the cells were transferred into 4-well LabTek Chamber Slide for $1 \mathrm{hr}$ before imaging. A final concentration of $150 \mathrm{ng} / \mathrm{ml}$ EGF was added to induce Rac activity. Rac FRET probe was kindly provided by Dr. Erez Raz. FRET experiment in S2 cells were carried out on Olympus IX81 microscope using 40X, 1.3 numerical aperture oil immersion objective. CFP and YFP signals were recorded using Chroma 86002BS dichroic mirror sets: CFP (excitation, 436/10nm; emission, 470/30nm), YFP(excitation, 436/10nm; emission, 535/30nm). A 25\% neutral density filter was used to reduce bleaching.

FRET images of live cultured egg chambers were acquired with Zeiss LSM710 microscope. $458 \mathrm{~nm}$ laser was used to excite the sample. CFP and YFP emission signals were collected 
through Channel I (470-510 nm) and Channel II (525-600 nm) respectively. To capture single, high-resolution, stationary images, 40X/1.1 water immersion objective was used. CFP and YFP images were acquired simultaneously for most of the experiments. Sequential acquisition of CFP and YFP channels with alternative orders were tested and gave the same result as simultaneous acquisition. CFP and YFP images were first processed by ImageJ software. A background ROI was subtracted from the original image. The YFP images were registered to CFP images by TurboReg pulgin. Gaussian smooth filter was then applied to both channels. The YFP image was thresholded and converted to binary mask with background set to zero. Final ratio image was generated by MATLAB program, during which only the unmasked pixel was calculated and all YFP/CFP ratios were adjusted to the initial FRET ratio to reduce the effect of bleaching. FRET images were analyzed using MATLAB. Border cell cluster was first isolated with its center calculated basing on its contour. Then the cluster was divided into 30 sectors, each of which occupies a 12-degree central angle. Because the center of the cluster contains the polar cells which do not express slbo-Gal4 and therefore were devoid of signal, only the signal within the distal 1/3 of each sector from the center was calculated. Average signal of each sector become a vector of length 31 . The first and last element corresponding to the -15 and 15 sectors were the same, so the front of border cell was centered at zero. A heatmap was composed by 30 vectors from different egg chambers with the same genotype. All vectors for each genotype were further averaged and smoothed to generate a representative curve of the FRET distribution around the cluster.

Measurement of migration speed, protrusion number, directionality index and protrusion density.

The distance of the center of the border cell cluster between the first and last time points in a time lapse series was measured in Imaris software. This distance divided by the elapsed time gave the speed. Cell protrusions were counted as follows: a circle corresponding to the average cluster diameter was drawn and any extension more than $2 \mu \mathrm{m}$ beyond that was considered a protrusion. The directionality index (DI) was calculated using the following equation:

$$
D I=\left(\sum_{i=1}^{N} \vec{p}_{i} \cdot \vec{d}\right) / \sum_{i=1}^{N}\left\|\vec{p}_{i}\right\|
$$

where $\mathrm{N}$ is the total number of major protrusions, $\vec{p}_{i}$ is the $i$ th protrusion vector, and $\vec{d}$ is the unit vector of migration direction. Protrusion vector is calculated by fitting the major protrusion by a parabola whose peak together with the cluster center gives the vector's direction and length. Protrusion density was generated by dividing the number of all the recognizable membrane protrusions by the estimated cell perimeter in micrometers. The morphology analysis and quantification were done in MATLAB.

\section{Immunohistochemistry}

Drosophila ovaries were dissected and fixed as described previously 31 and incubated with 1.4 units Alexa 488-conjugated phalloidin (Molecular Probes) per ml and $1 \mu \mathrm{g} / \mathrm{ml}$ DAPI 
prior to imaging on a Zeiss 510-Meta confocal microscope and 3D reconstruction using Imaris software.

\section{Supplementary Material}

Refer to Web version on PubMed Central for supplementary material.

\section{Acknowledgements}

This work was supported by GM046425 to D.J.M and GM 057464 to K.M.H. and by the Cell Migration Consortium.

\section{References}

1. Ridley AJ, Paterson HF, Johnston CL, Diekmann D, Hall A. The small GTP-binding protein Rac regulates growth factor-induced membrane ruffling. Cell. 1992; 70:401-410. [PubMed: 1643658]

2. Sander EE, Collard JG. Rho-like GTPases: their role in epithelial cell-cell adhesion and invasion. Eur J Cancer. 1999; 35:1302-8. [PubMed: 10658518]

3. Fukata M, Kaibuchi K. Rho-family GTPases in cadherin-mediated cell-cell adhesion. Nat Rev Mol Cell Biol. 2001; 2:887-97. [PubMed: 11733768]

4. Friedl P, Gilmour D. Collective cell migration in morphogenesis, regeneration and cancer. Nat Rev Mol Cell Biol. 2009; 10:445-57. [PubMed: 19546857]

5. Weijer CJ. Collective cell migration in development. J Cell Sci. 2009; 122:3215-23. [PubMed: 19726631]

6. Rorth P. Collective cell migration. Annu Rev Cell Dev Biol. 2009; 25:407-29. [PubMed: 19575657]

7. Bidard FC, Pierga JY, Vincent-Salomon A, Poupon MF. A "class action" against the microenvironment: do cancer cells cooperate in metastasis? Cancer Metastasis Rev. 2008; 27:5-10. [PubMed: 18066649]

8. Wu YI, et al. A genetically encoded photoactivatable Rac controls the motility of living cells. Nature. 2009; 461:104-8. [PubMed: 19693014]

9. Silver DL, Montell DJ. Paracrine signaling through the JAK/STAT pathway activates invasive behavior of ovarian epithelial cells in Drosophila. Cell. 2001; 107:831-41. [PubMed: 11779460]

10. Silver DL, Geisbrecht ER, Montell DJ. Requirement for JAK/STAT signaling throughout border cell migration in Drosophila. Development. 2005; 132:3483-92. [PubMed: 16000386]

11. Bai J, Uehara Y, Montell DJ. Regulation of Invasive Cell Behavior by Taiman, a Drosophila Protein Related to AIB1, a Steroid Receptor Coactivator Amplified in Breast Cancer. Cell. 2000; 103:1047-1058. [PubMed: 11163181]

12. Duchek P, Rorth P. Guidance of cell migration by EGF receptor signaling during Drosophila oogenesis. Science. 2001; 291:131-3. [PubMed: 11141565]

13. Duchek P, Somogyi K, Jekely G, Beccari S, Rorth P. Guidance of cell migration by the drosophila pdgf/vegf receptor. Cell. 2001; 107:17-26. [PubMed: 11595182]

14. McDonald JA, Pinheiro EM, Kadlec L, Schupbach T, Montell DJ. Multiple EGFR ligands participate in guiding migrating border cells. Dev Biol. 2006; 296:94-103. [PubMed: 16712835]

15. McDonald JA, Pinheiro EM, Montell DJ. PVF1, a PDGF/VEGF homolog, is sufficient to guide border cells and interacts genetically with Taiman. Development. 2003; 130:3469-78. [PubMed: 12810594]

16. Wang X, Adam JC, Montell D. Spatially localized Kuzbanian required for specific activation of Notch during border cell migration. Dev Biol. 2007; 301:532-40. [PubMed: 17010965]

17. Murphy AM, Montell DJ. Cell Type-specific Roles for Cdc42, Rac, and RhoL in Drosophila Oogenesis. JOURNAL OF CELL BIOLOGY. 1996; 133:617-630. [PubMed: 8636236]

18. Geisbrecht ER, Montell DJ. A role for Drosophila IAP1-mediated caspase inhibition in Racdependent cell migration. Cell. 2004; 118:111-25. [PubMed: 15242648] 
19. Prasad M, Jang ACC, Montell D. A Protocol for culturing Drosophila melanogaster egg chambers for live imaging. Nature Protocols in preparation. 2007

20. Prasad M, Montell DJ. Cellular and Molecular Mechanisms of Border Cell Migration Analyzed Using Time-lapse Live-cell Imaging. Developmental Cell. 2007; 12:997-1005. [PubMed: 17543870]

21. Montell DJ. Border-cell migration: the race is on. Nat Rev Mol Cell Biol. 2003; 4:13-24. [PubMed: 12511865]

22. Jang AC, Chang YC, Bai J, Montell D. Border-cell migration requires integration of spatial and temporal signals by the BTB protein Abrupt. Nat Cell Biol. 2009; 11:569-79. [PubMed: 19350016]

23. Montell DJ, Rorth P, Spradling AC. slow border cells, a locus required for a developmentally regulated cell migration during oogenesis, encodes Drosophila C/EBP. Cell. 1992; 71:51-62. [PubMed: 1394432]

24. Llense F, Martin-Blanco E. JNK signaling controls border cell cluster integrity and collective cell migration. Curr Biol. 2008; 18:538-44. [PubMed: 18394890]

25. Kardash E, et al. A role for Rho GTPases and cell-cell adhesion in single-cell motility in vivo. Nat Cell Biol. 12:47-53. sup pp 1-11. [PubMed: 20010816]

26. Rorth P. Collective guidance of collective cell migration. Trends Cell Biol. 2007; 17:575-9. [PubMed: 17996447]

27. Rorth P, et al. Systematic gain-of-function genetics in Drosophila. Development. 1998; 125:104957. [PubMed: 9463351]

28. Lee T, Luo L. Mosaic analysis with a repressible cell marker for studies of gene function in neuronal morphogenesis. Neuron. 1999; 22:451-61. [PubMed: 10197526]

29. Edwards KA, Demsky M, Montague RA, Weymouth N, Kiehart DP. GFP-moesin illuminates actin cytoskeleton dynamics in living tissue and demonstrates cell shape changes during morphogenesis in Drosophila. Dev Biol. 1997; 191:103-17. [PubMed: 9356175]

30. Luo L, Liao YJ, Jan LY, Jan YN. Distinct morphogenetic functions of similar small GTPases: Drosophila Drac1 is involved in axonal outgrowth and myoblast fusion. Genes and Development. 1994; 8:1787-1802. [PubMed: 7958857]

31. McDonald JA, Montell DJ. Analysis of cell migration using Drosophila as a model system. Methods Mol Biol. 2005; 294:175-202. [PubMed: 15576913] 


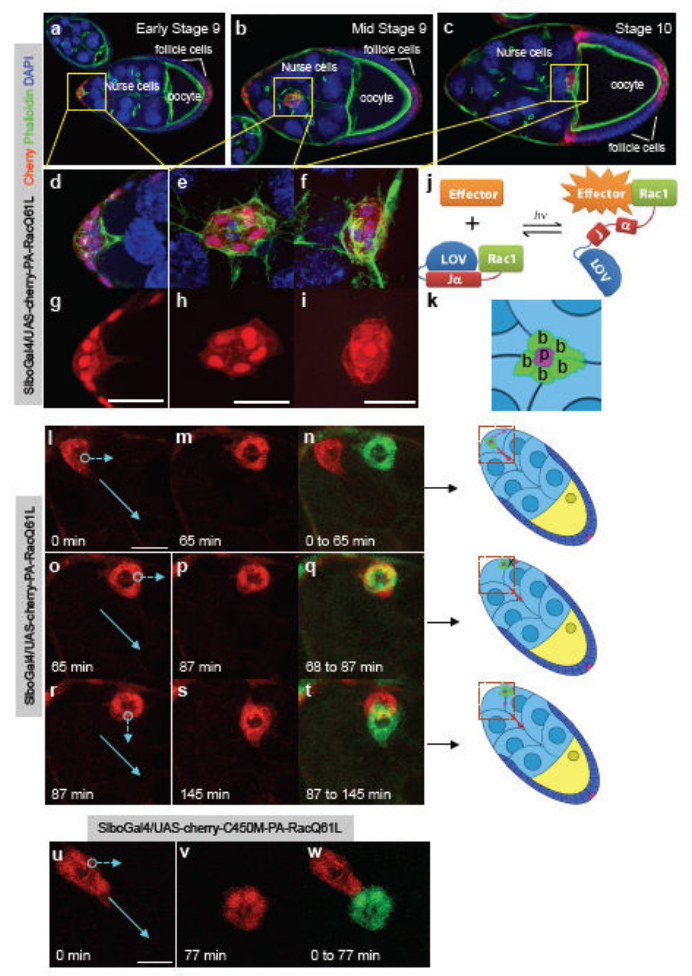

Figure 1.

Local activation of PA-Rac1 redirects an entire border cell group.

(a-c) Egg chambers labelled with DAPI (blue) to stain all nuclei, Alexa 488-phalloidin (green) to mark actin filaments, and mCherry (red) to show PA-RacQ61L. (d-f), Higher magnification views of border cells from each stage. (g-i) PA-RacQ61L expression only. (j) Schematic diagram from8 showing the mechanism of PA-Rac light-activation. (k) Schematic of border cell cluster composed of two non-migratory polar cells (purple, $\mathrm{p}$ ) which do not express slbo-Gal4 and are therefore unlabeled in all subsequent images. Polar cells are surrounded by 4-6 migratory border cells (green, b). (l-t) Selected still images from a time-lapse film of the response of border cells to photoactivation of PA-RacQ61L. (I-n) Photoactivation diverts border cells to the edge of the egg chamber. (o-q) Continued photoactivation in same direction did not move them further along the edge. (r-t) Photoactivation of the same cluster in a different position drove movement towards the egg chamber center. In $\mathbf{n}, \mathbf{q}$ and $\mathbf{t}$ the starting position of the cluster is shown in red and the final position in green. Schematics at right show the position of the treated cluster within the egg chamber. Red boxes indicate the regions shown in the micrographs. Red arrow indicates the normal direction of migration. Pink arrow shows the direction the cells move if they respond to the light. (u-w) Phototreatment of light insensitive control C450M-PA-Rac1Q61L. In l, n, $\mathbf{q}$ and $\mathbf{u}$, solid arrows indicate the normal direction of migration; circles indicate where the laser light was applied. Dashed arrows indicate the direction the cells move if they respond to the light. Scale bars, $20 \mu \mathrm{m}$. 

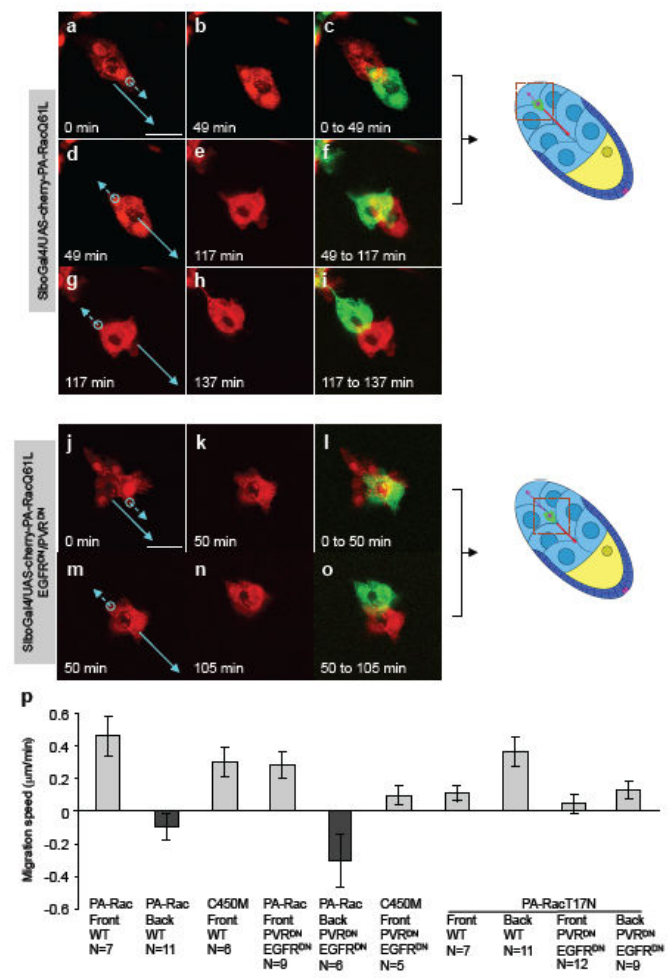

Figure 2.

Forward or backward movement in response to photoactivatable Rac.

(a-i) In an otherwise wild-type background, PA-RacQ61L can promote forward (a-c) or backward (d-i) movement. (j-l) Forward and (m-o) reverse migration of border cells expressing $\mathrm{PVR}^{\mathrm{DN}}, \mathrm{EGFR}^{\mathrm{DN}}$, and PA-RacQ61L. The schematics at the right show the position of the cluster within the egg chamber. Scale bars, $20 \mu$. In panels with two colors, red represents the starting position and green shows the ending position over the indicated time period. p) Average migration speeds for clusters expressing the indicated proteins in response to illumination of the front or the back of the cluster. PA-Rac refers to PARacQ61L. C450M is the light-insensitive control. Values represent the average of the indicated number $(\mathrm{N})$ of experiments and error bars show the standard deviation. 

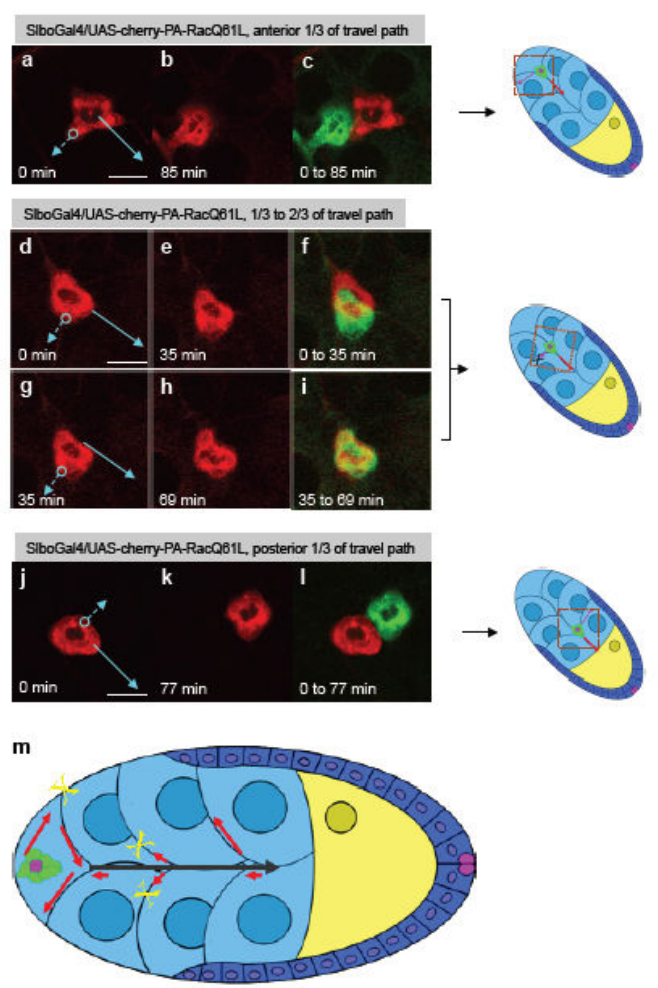

Figure 3.

Responsiveness of border cells to PA-RacQ61L depends on their location within the egg chamber.

(a-c) Within the anterior third of the egg chamber, photoactivation diverts border cells. (d-i) In the middle third, photoactivation has little effect. (c) In the posterior third,

photoactivation again drives border cells toward the side. Scale bars represent $20 \mu \mathrm{m}$, elapsed time is shown in minutes. Schematics show border cell position within the egg chamber. In panels with two colors, red indicates the starting position and green shows the ending position. (m) Summary of experiments. The lengths of the arrows indicate the average distance migrated in the indicated direction in response to PA-RacQ61L, for border cells starting at the base of the arrow. The black arrow indicates the normal migration direction. Yellow Xs indicate positions beyond which border cells did not move. Each arrow summarizes at least five experiments. 

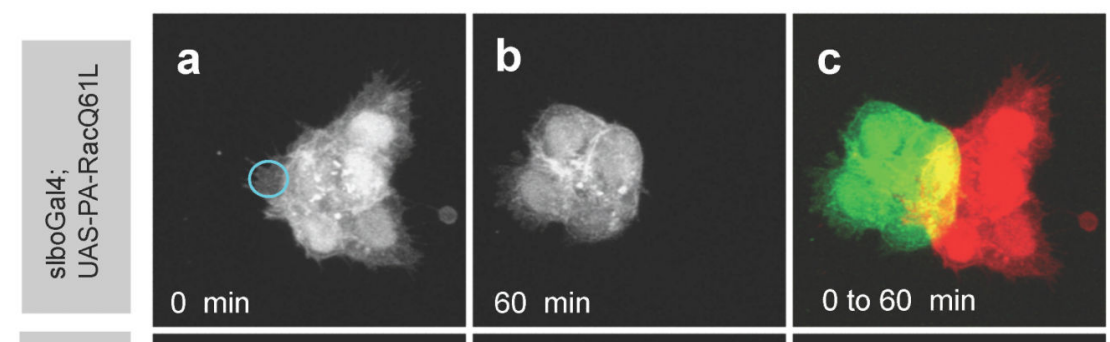

\# of Cells with Protrusion
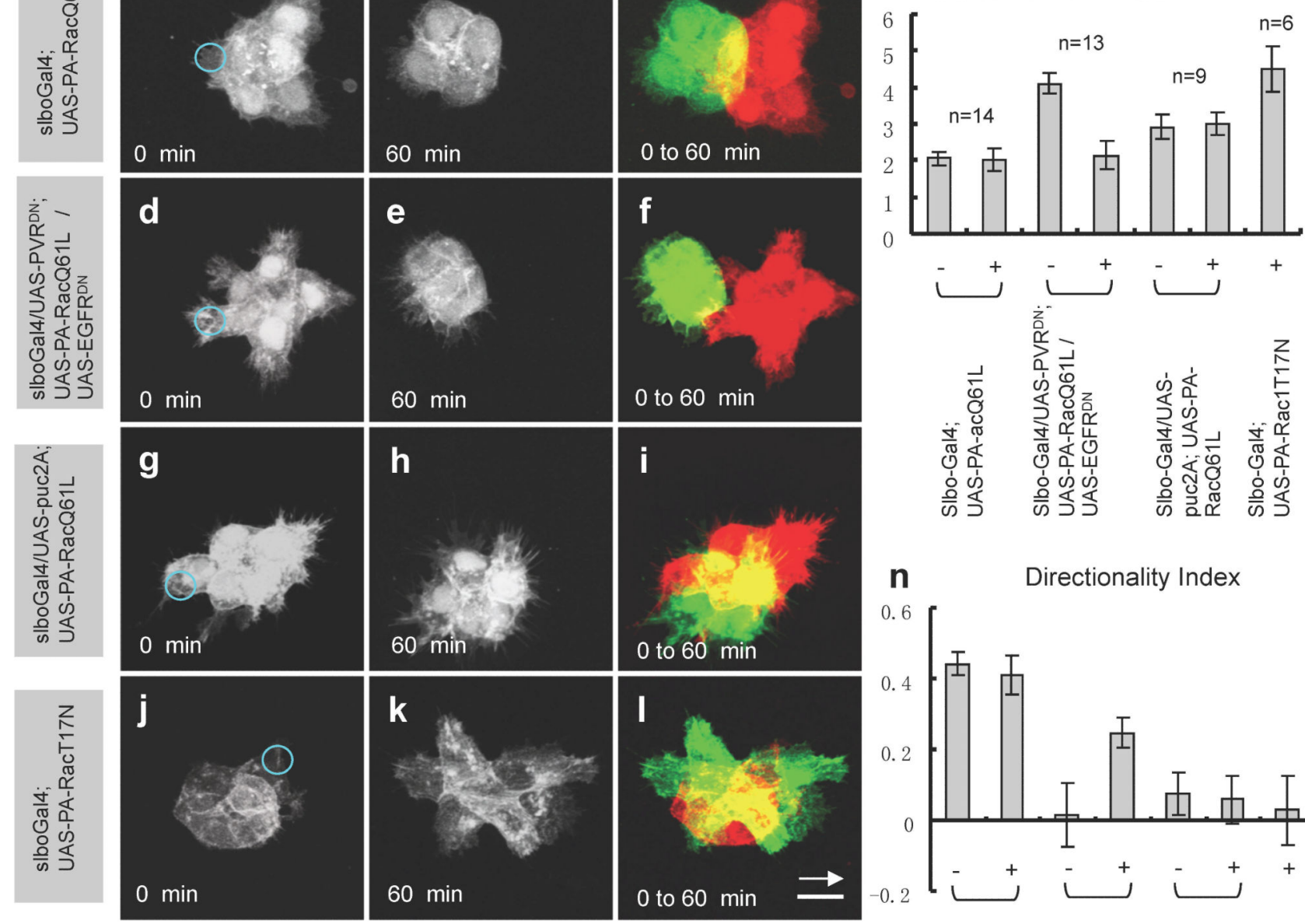

Figure 4.

Local photoactivation or photoinactivation of Rac in one cell affects the morphology and behavior of other cells in the group.

(a-l) Confocal images of border cell clusters before ( $0 \mathrm{~min})$ and after $(60 \mathrm{~min})$ photoactivation. Circles indicate areas of laser treatment. The white arrow in panel $\mathbf{l}$ indicates the direction the border cells would normally migrate and applies to all panels. Scale bar is $10 \mu \mathrm{m}$. In $\mathbf{c}, \mathbf{f}, \mathbf{i}$ and $\mathbf{I}$, red shows the starting position and green shows the ending position. $\mathbf{m}$, The average number of cells sending protrusions simultaneously within one cluster was calculated from 3-D reconstructed images (see methods and Figure S6). "-” and "+" indicate before and after photoactivation. n, Directionality indices were calculated from the same samples (see methods). 

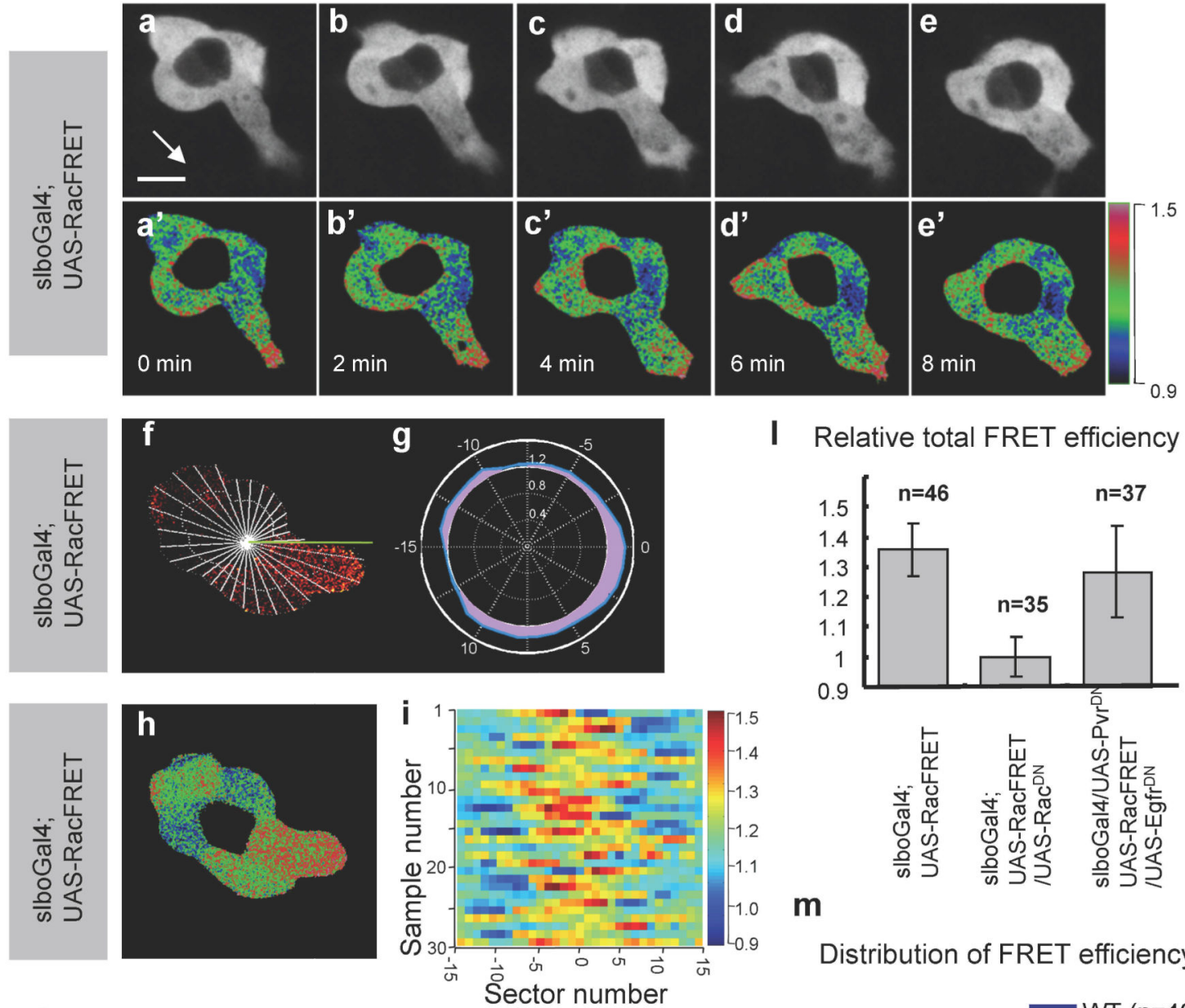

I Relative total FRET efficiency
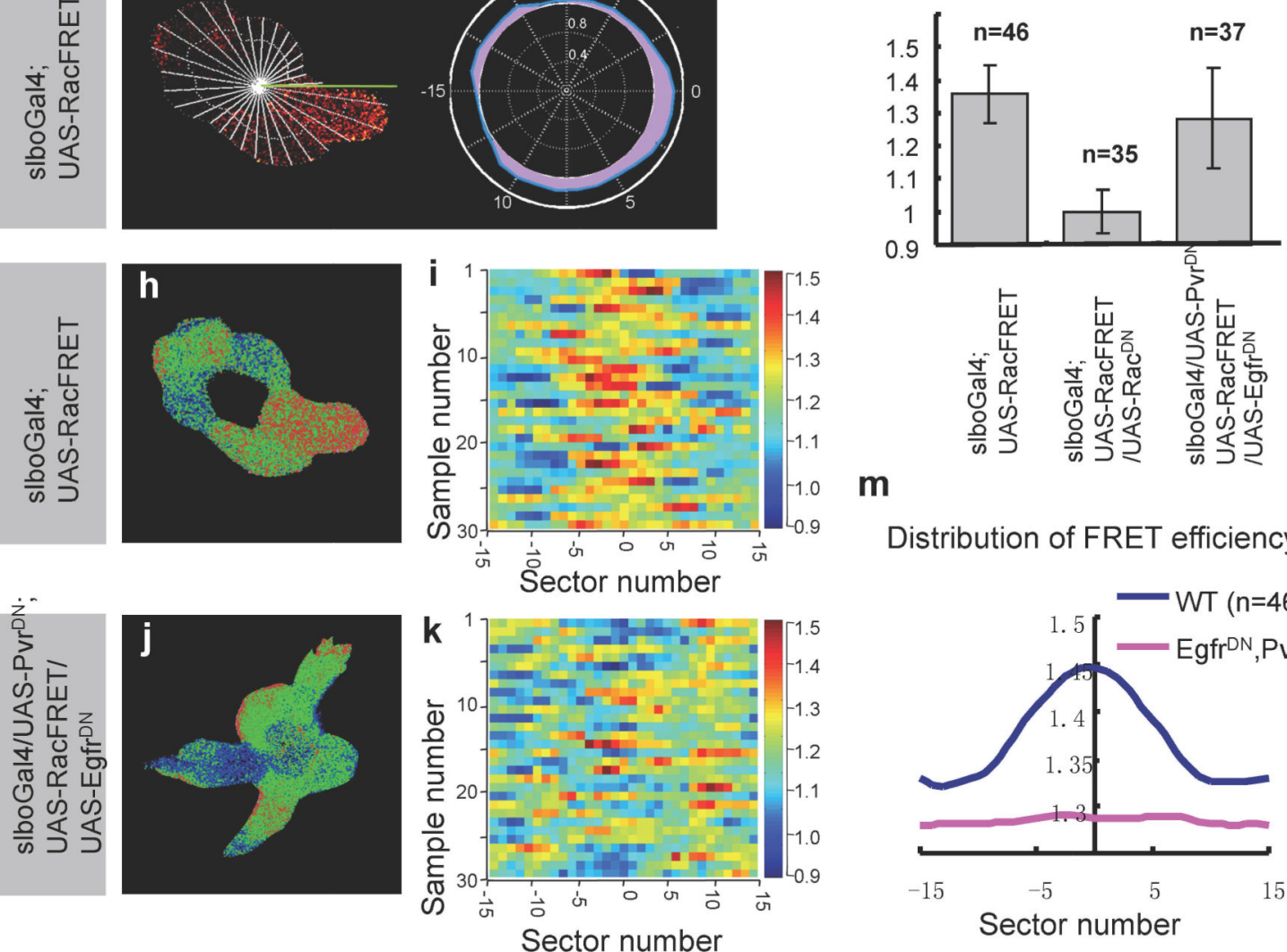

m

Distribution of FRET efficiency

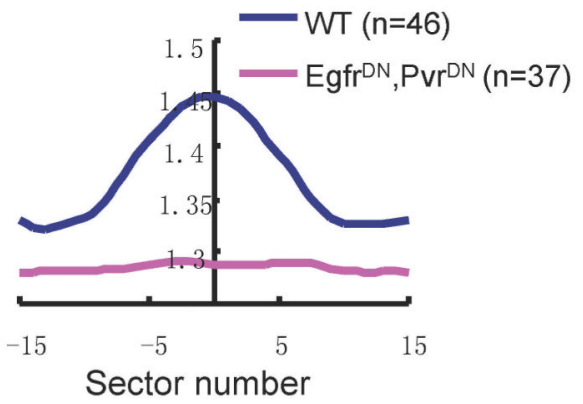

Figure 5.

Rac activity pattern in migrating border cell clusters.

(a-e') A time lapse series of migrating border cells. (a-e) YFP channel only (a'-e') processed FRET signal f. FRET image of wild-type border cells displayed with Red-Hot pseudocolor divided into 30 sectors. The yellow line shows the direction of migration. The white circle indcates the central region that was excluded from the analysis. (g) Average FRET efficiency from image $\mathbf{f}$ plotted in a radar map. Efficiency higher than 1.2 is highlighted in purple. (h-j) Representative FRET patterns in wild-type (h) and EGFR ${ }^{\text {DN }}$ and $\mathrm{PVR}^{\mathrm{DN}}$-expressing (j) border cells. (i, k) Heatmaps from 30 examples of each genotype. 
Each row represents the FRET signal distribution of an individual border cell cluster. Positions from -15 to 15 plotted on the $\mathrm{x}$-axis correspond to the sectors, where 0 represents the front of the cluster. I. FRET efficiencies in border cells of the indicated genotypes. All results were normalized to the efficiency of $\operatorname{Rac}^{\mathrm{DN}}$. (m) Distributions of average FRET efficiencies in wild-type (blue) and $\mathrm{PVR}^{\mathrm{DN}} / \mathrm{EGFR}^{\mathrm{DN}}$ border cells, plotted as a function of sector number, where 0 represents the front. 